\title{
Melatonin Ameliorates Burn-Induced Liver Injury by Modulation of Nrf2 and Nf- kB Signaling Pathways
}

\author{
Ganka Bekyarova ${ }^{1 *}$, and Maria Tzaneva ${ }^{2}$ \\ ${ }^{1}$ Divison of Pathophysiology, Medical University, Varna, Bulgario \\ ${ }^{2}$ Department of General and Clinical Pathology, Medical University, Varna, Bulgaria
}

Received: May 11, 2015; Accepted: May 26, 2015; Published: June 18, 2015

*Corresponding author: Ganka Bekyarova, Head, Department of Pathophysiology, Medical University of Varna, 55Marin Drinov St, BG-9002 Varna, Bulgaria, E-mail: gabekyarova2001@yahoo.com

\begin{abstract}
Absract
Severe thermal injury is a common traumatic injury that results in local tissue damage and systemic response and represents a serious clinical problem. A skin burn leads to dysfunction of organs distant from the original burn skin injury, including the liver. The pathophysiology of burn-induced liver injury is very complicated and remains unclear. Increasing evidence implicate activation of the inflammatory response along with the oxidative stress as the main mechanisms of hepatic injury in burns. Melatonin is a multifunctional molecule that might counteract all pathophysiologic steps and displays beneficial effects against cellular damage, induced by thermal injury. This review summarises protective effect of melatonin on hepatic injury related with suppression of oxidative stress, inflammatory response and apoptosis induced by thermal skin injury. Special emphasis is focused on modulating effect of melatonin on Nuclear Erythroid 2-related factor 2 (Nrf2) and Nuclear Factor-kappa B (NF$\mathrm{kB}$ ) signaling pathways, related with activation of cellular defense against oxidative stress and reduction of proinflammatory mediator production. The overall findings suggest that melatonin should be exploited as a therapeutic tool to prevent or reverse the harmful effects of thermal trauma.
\end{abstract}

Keywords: NF-kB; Nrf2; Oxidative stress; Inflammation; Melatonin; Liver injury; Burns

\section{Introduction}

A severe burn is a devastating injury affecting every organ system and leads to complications with poor outcome. Liver plays an important role in metabolism, inflammatory response, homeostasis and host defense mechanisms. It is also a major organ responsible for initiating Multi-Organ Dysfunction Syndrome (MODS) following burn injury [1,2]. The pathophysiology of burn-induced liver injury is complex and not well understood. In this regard, several pathogenetic mechanisms have been proposed in particular ischemia/reperfusion, generation of lipid metabolites, cytokine overproduction, depletion of glutathione and mitochondrial dysfunction These complex mechanisms cause liver dysfunction, damage of hepatic parenchyma and, eventually, cell death [3-5].

Taking into consideration the role of the lipid peroxidation in burn-induced hepatic injury, the interference with hepatic oxidative status and the inflammatory response, it is logical to hypothesize that therapy with substances possessing antioxidant, anti-inflammatory and anti-apoptotic effects may be beneficial in reducing these complications.

Melatonin (N-acetyl-5-methoxytryptamine) is secreted in the pineal gland and extra pineal tissues. Melatonin possesses a wide variety of biological effects such as chronobiological, sedative and anxiolytic. Melatonin may act as an antioxidant and a potent scavenger of both oxygen and nitrogen reactive molecules. The free radical scavenging activity of melatonin is receptor independent process whereas the indirect effect likely involves specific receptors [6]. Melatonin stimulates the activity of enzymes involved in antioxidant defense and reduces the production of proinflammatory mediators $[7,8]$. Melatonin is a small, highly lipophilic and hydrophilic molecule. It passes freely through membranes and distributes in all subcellular compartments [5]. These evidences suggest that these pleiotropic functions of melatonin may be used clinically under conditions where its circulating levels are reduced $[9,10]$. Because of its antioxidant, anti-inflammatory and anti-apoptotic effects, exogenous melatonin attenuates ischemia/reperfusion and hepatotoxins induced liver injury [11-13].

\section{Antioxidative and anti-inflammatory effect of melatonin}

Melatonin participates in the regulation of important physiological processes. This hormone is known to regulate the circadian rhythms induced by a short light dark cycle and the circadian rhythms [14,15]. A number of studies demonstrate that melatonin is an interesting pharmacological agent in the treatment of inflammatory conditions [8-16]. The beneficial effect of melatonin in these studies is associated with: 1) antioxidant effect of this indole and 2) modulation of the inflammatory response.

A large body of evidence indicates that melatonin is a major Scavenger of Radical Oxygen Species (ROS) and Reactive nitrogen (RNS species and activator of antioxidant enzymes [17-19]. Under normal conditions ROS and RNS are produced in mitochondria and they are neutralized by non enzymatic 
scavengers and antioxidant enzymes. Melatonin, vitamins C and $\mathrm{E}$, reduced glutathione and uric acid are non enzymatic scavengers [20,21]. Antioxidant enzymes such as Superoxide Dismutase (SOD), Catalase (CAT), Glutathione Peroxidase (GPX), or Glutathione Reductase (GR) are important in the defense against the damaging effect of ROS and RNS [17,22]. Oxidative stress occurs as a result of an imbalance between the excessive production of ROS and RNS and impaired ability of tissue to detoxify free radical in burns. Accumulated ROS and RNS in tissues have direct toxic effect on cells: damage nuclear DNA membrane lipids and cytosolic proteins [23-25]. The harmful effects of ROS and RNS have been shown in experimental thermal trauma [5,9]. These alterations often results in the production of more ROS and lipid peroxides. This leads to cellular dysfunction and/or cell death. Cells possess defense antioxidant system that protect against oxidative stress and suppress oxidative injury. Such antioxidants as glutathione can scavenge ROS and restrict cellular alteration. Activation of antioxidant enzymes is an adaptive defense mechanism against oxidative stress.

Nuclear Factor erythroid 2-related factor (Nrf2) is a transcription factor activated by oxidative stress that has an important role in regulation of the expression of detoxifying and antioxidant genes. Activated Nrf2 translocates to nucleus, binds to Antioxidant Response Element (ARE), and enhances the up-regulation of a network of cytoprotective enzyme such as Heme Oxygenase-1 (HO-1), Glutamate Cysteine Ligase (GCL), thioredoxin reductase, glutathione-S-transferase and NAD(P) H:quinone oxidoreductase that mediate cell survival [26]. Other Antioxidant Enzymes (SOD and CAT) and non-enzymatic scavengers such as glutathione are also involved in scavenging ROS. Nrf2/ARE signaling plays an important role in the cell protection from ROS. Nrf2/ serve as a novel target for designing therapy to prevent and treat diseases in which oxidative stress is involved. In addition, Nrf2 signaling pathway can attenuate proinflammatory stimuli leading to restrict inflammatory injury $[26,27]$.

ROS also activate the transcription factor NF-kB and inflammatory signaling pathways leading to increased production of inflammatory mediators [28]. NF-kB is sequestered in the cytoplasm of cells, bound to its inhibitor IkB. NF-kB is activated by degradation of this inhibitor by ROS, proinflammatory cytokines and bacterial products. After liberation, NF-kB translocates to the nucleus binding to DNA and enhances the expression genes of pro inflammatory cytokines (tumor necrosis factor- $\alpha$ (TNF- $\alpha$ ), interleukins (IL-1 $\beta$, IL-6, IL-8), adhesion molecules (VCAM-1, ICAM-1), inducible nitric oxide synthase (iNOS) and cyclooxygenase (COX) [29,30]. Therefore, therapeutic agents that inhibit the activation of NF-kB-mediated inflammatory response may be an attractive strategy for limiting the damaging effect of the proinflammatory mediators under pathological conditions.

Recently, melatonin has been shown to activate signal transduction pathways leading to the activation of antioxidant enzymes and to the reduction of inflammatory mediators [16]. Melatonin upregulates the expression of Nrf2, involved in the induction of antioxidant enzymes such as SOD, CAT, GPx, GST and
GR [7]. Since, melatonin is known to activate these antioxidant enzymes. It is possible that antioxidant effect of melatonin is mediated by Nrf2 transcriptional factor $[13,16]$.

Melatonin modulates the NF- $\kappa \mathrm{B}$ inflammatory signaling pathway. Melatonin plays a key role in the induction of antiinflammatory cytokine and suppression and pro-inflammatory signaling [16]. It reduces gene expression and synthesis of proinflammatory cytokine such as TNF- $\alpha$, IL- $1 \beta$, IL-6, IL-8 and prostaglandins $[31,32]$. In addition, melatonin is also reported to modulate the processes of apoptosis and necrosis, to stimulate the production of Vascular Endothelial Growth Factor (VEGF), and to activate the process of angiogenesis [33].

Melatonin upregulates Nrf2 expression and increases antioxidant activities, reduces $\mathrm{NF}-\kappa \mathrm{B}$ activation and inhibits iNOS and pro-inflammatory cytokine expression in rats with dimethylnitrosamine (DMN)-induced liver injury [14]. Melatonin could modulate inflammation by decreasing NF- $\kappa B$ activation and oxidative stress by increasing Nrf2 expression that might be related, at least in part, with its hepatoprotective effect.

\section{Hepatoprotective effect of melatonin in thermal skin injury}

Burns initiate Systemic Inflammatory Response Syndrome (SIRS) where the generation of pro-inflammatory cytokines and free radicals causes a progressive distant organs dysfunction $[1,3,34,35]$. Experimental studies have shown that application of melatonin protects liver tissue against the damage caused by thermal trauma [6,7]. Melatonin based on its small molecular size and high lipophilic capacity reduces liver injury in burn rat model $[33,36]$ and other models of oxidative stress $[15,28,37,38]$.

Overproduction of free radicals along with unbalanced hepatic antioxidant defense capacity initiates lipid peroxidation and directly damages plasma and intracellular membranes which alters membrane-bound proteins. [33,36,39]. Both ROS and RNS enhance the production of reactive aldehydes such as 4-HNE with potent pro-inflammatory and pro-apoptotic properties [40]. Increased levels lipid peroxidation and oxidative injury is demonstrated by increased expression of 4-HNE in liver in burn rat model (Figure 1). The activation of peroxidative mechanisms results in cellular dysfunction, apoptosis or necrosisin hepatocytes with the release of the hepatic enzymes into circulation. Serum Aminotransferase (ALT and AST) activities increases markedly. Early postburn and these elevations attenuate with melatonin $(10 \mathrm{mg} / \mathrm{kg})$, thus indicating melatonin's role in burn-induced hepatoprotection.

Several studies associated with an increased antioxidant activity shows to counteract the oxidative stress-induced by thermal injury $[41,42]$. CuZnSOD expression as front-line antioxidant increases as compensatory reaction early post-burn when oxidative stress increases [43,44]. HO-1 is the inducible isoform of heme oxygenase, and it is reported to protect against liver injuries, too [45]. Our results shows that HO-1 protein is expressed in hepatocytes surrounding the central vein in liver (Figure 2). 

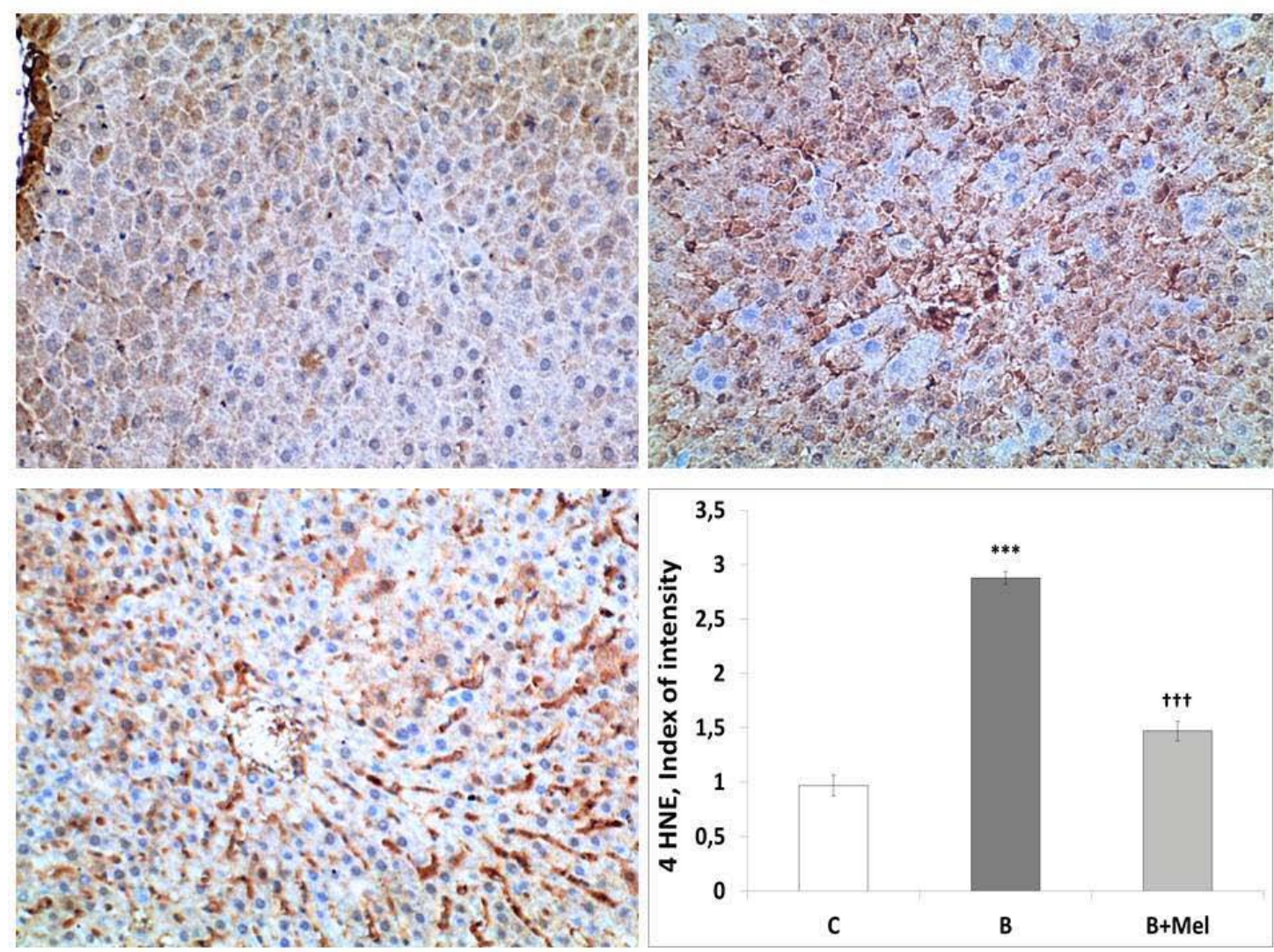

Figure 1: Effect of melatonin on the expression of 4-hydroxy-nonenal in liver after thermal trauma

C-controls; B-burned non treated; B + Mel- burned treated with melatonin

${ }^{* * *} p<0.001$ - B vs $\mathrm{C} ;+++p<0.001-\mathrm{B}+$ MelvsB

Immunohistochemistry detection for 4-hydroxynonenal (4-HNE) in liver

A: It is localized in both Sinusoidal Endothelial Cells (SECs) and hepatocytes in the control group. The staining intensity of 4-HNE positive cells was weak.

B: In the burned group, induction of 4-HNE positive cells was principally in the sinusoidal cells and mainly in hepatocytes around the central vein. It was moderate to strong in the individually cells.

C: In the burned group reacted with melatonin, induction of 4-HNE positive cells was principally in SEC and some hepatocytes around the central vein. The number of 4-HNE positive cells was lower than in the burned non treated. (Immunohistochemsitry, x 400).

Indeed, HO-1 expression and levels of products of lipid peroxidation (MDA and 4-HNE) in liver were elevated within 24 $\mathrm{h}$ after burns [46]. Because H0-1 is induced by oxidative stress, it could be speculated that the increased expression of HO-1 in liver is likely related to overproduction of ROS/RNS after burns. Melatonin reduces the levels of lipid peroxidation productes, improves liver histopathological changes and augmented the increase of HO-1 expression in burned rats [47]. These findings suggest that the protective mechanism of melatonin against burn-induced liver injury could be closely related with HO-1 overexpression and increased cellular antioxidant capacity. Both endogenous HO-1 and melatonin-induced HO-1 may enhance the overall cellular antioxidant capacity and prevent oxidative stress induced cytotoxicity in liver after burns. Several studies have reported that $\mathrm{HO}-1$ exerts protective effect against a variety of pathological conditions, such as ischemia/ reperfusion, endotoxin, hepatotoxins $[37,38]$. The cytoprotective effect of HO-1 under pathological conditions and oxidative stress depends on biliverdin, a free radical scavenger or CO anti-inflammatory anti apoptotic gas, both of which are degradation products of heme catabolized by HO-1 $[48,49]$.

Stress-activated Nrf2 transcription factor plays predominant role and mediates potent HO-1 and SOD activation. Melatonin increases $\mathrm{Nrf} 2$ expression and it is even more intensive in the cytoplasm of hepatocytes, but it is also present in the nuclei of endothelial cells after burns (Figure 3). Nrf2 is localized in the cytoplasm bound to Keap1, a cytoskeleton-associated protein. A possible mechanism by which melatonin increases Nrf2 expressionis related to the dissociation of Nrf2/Keap1 (repressor) and translocation of Nrf2 to the nucleus, where it binds to ARE in the promoter of the genes involved in the antioxidant protection [50]. Melatonin may ameliorate burn-induced liver injury through inhibition of free-radical activating lipid peroxidation and elevation of expression of the transcription factor Nrf2 and some antioxidant enzymes such as HO- 1 and SOD. The activation of HO-1 and Nrf2 transcriptional factor is demonstrated in other models of hepatic and oxidative stress $[26,51,52,53]$. 

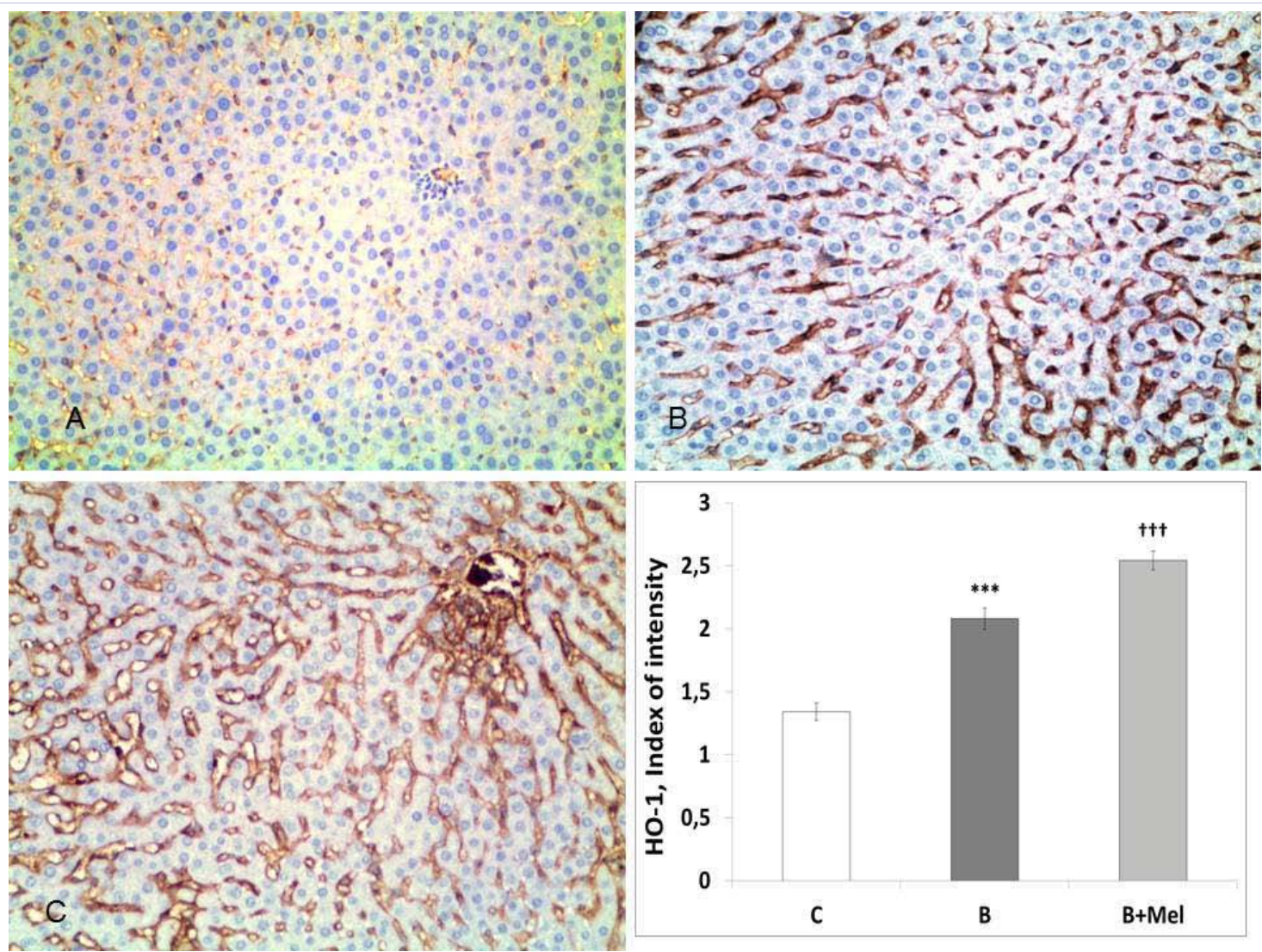

Figure 2: Effect of melatonin on the expression of Heme-oxygenase-1l in liver after thermal trauma

C-controls; B-burned non treated; B + Mel- burned treated with melatonin

*** $p<0.001$ - B vs $\mathrm{C} ;+++p<0.001-\mathrm{B}+$ Mel vs $\mathrm{B}$

Immunohistochemistry detection for heme-oxygenase-1 (HO-1) in liver

A: It is localized in SECs of the liver in the control group. The staining intensity of HO-1 positive cells was weak.

B: In the burned group, induction of HO-1 positive cells was in the sinusoidal cells. It was moderate to strong in the individually cells.

C: In the burned group treated with melatonin, induction of HO-1 positive cells was principally in the sinusoidal cells. The number of HO-1 positive cells was higher than in the burned non treated. (Immunohistochemsitry, $\mathrm{x} 400$ ).

Melatonin activates $\mathrm{Nrf} 2 / \mathrm{HO}-1$ signaling pathway and acts as a natural inducer of an antioxidant protection (Figure 4). The stimulation of protective mechanisms of the cells by activating Nrf2 transcription antioxidant factor is a new mechanism for protection against burn-induced liver damage. These results demonstrate that melatonin could induce the activation of HO-1/Nrf-2 pathway as a part of the hepatoprotective effects of melatonin in burns. Further research of the molecular mechanisms of the Nrf2 activation offers opportunities to develop a new therapeutic approach to therapy victims with thermal trauma.

Following a severe burn injury, the systemic inflammatory response encompasses the release of large quantities of proinflammatory cytokines such as IL-1 $\beta$, IL- 6 or TNF- $\alpha[1,54,55]$. The level of anti-inflammatory cytokines, such as IL-10, released in an attempt to counter-regulate the effects of the pro-inflammatory cytokines, decreases or remains unchanged $[56,57]$. The unbalance in pro-/anti-inflammatory cytokine production leads to alteration in function and structure of liver [3].
High TNF- $\alpha$ level may activate cell-death pathways and promotes the production of reactive oxygen species and lipid peroxidation and thus hepatic injury [29]. Furthermore, hepatocytes are much more sensitive to TNF- $\alpha$ and free radicals damaging effect when their antioxidant capacity is low. It appears that oxidative stress plays an important role in the primary cell and tissue destruction, and thus the secondary inflammatory reaction.

ROS/RNS may activate NF-kB transcription factor and increase the expression of pro-inflammatory cytokines. TNF $\alpha$ has a cytotoxic activity and may cause hepatic injury. On the other hand, increased production of TNF- $\alpha$ and free radicals may increase the expression of NF-kB transcription factor [58]. Probably, vicious cycle is formed between oxidative stress and inflammation by NF-kB activation. Such a positive relationship between markers of lipid peroxidation and inflammation in liver is found in other experimental models of thermal trauma [6].

Melatonin has the capability of scavenging both oxygen and nitrogen-based reactants including ONOO- and blocking NF-kB 

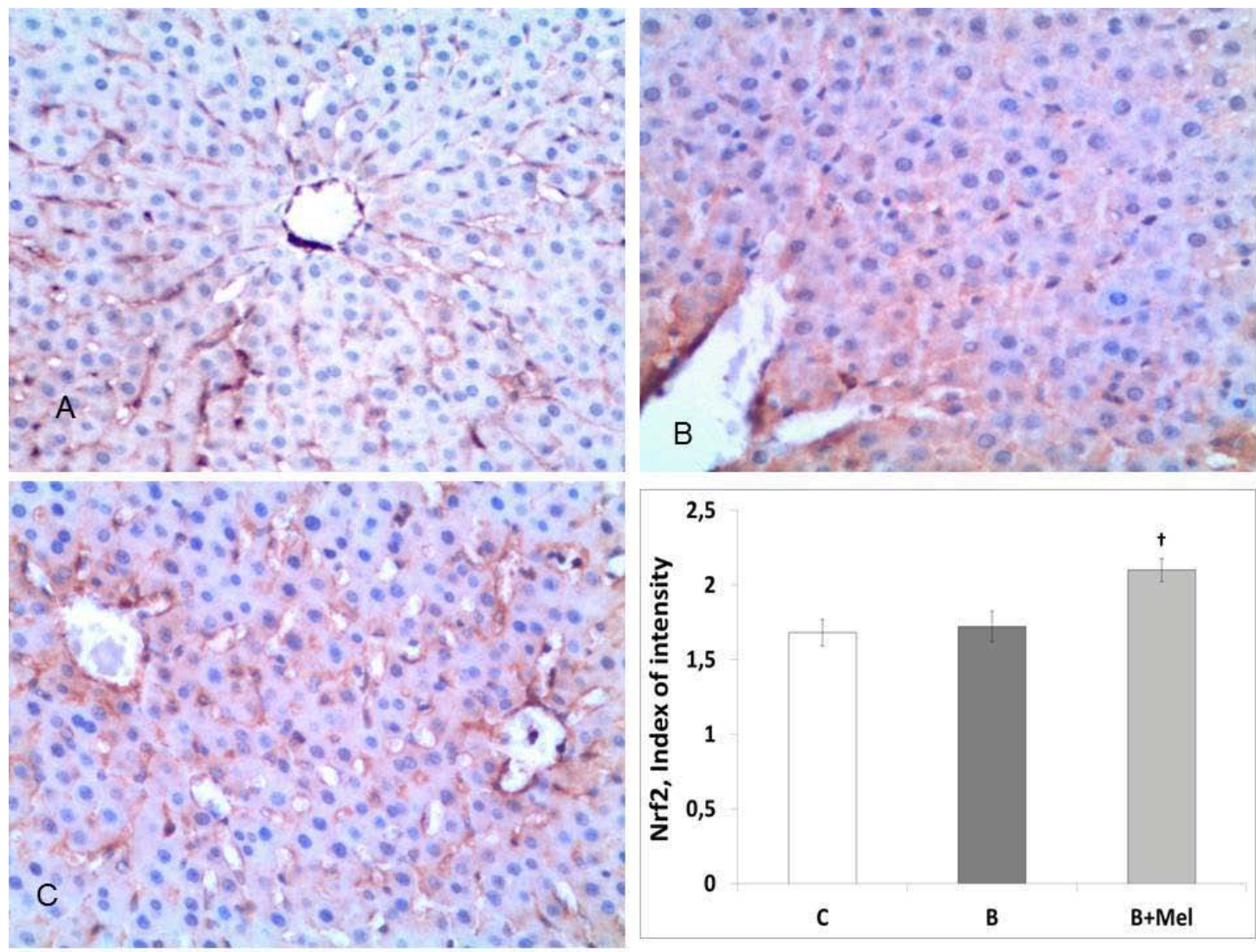

Figure 3: Effect of melatonin on the expression of Nrf2 transcriptional factor in liver after thermal trauma

$+p<0.001-\mathrm{B}+$ Mel vs B

Immunohistochemistry detection for of nuclear factor erythroid 2-related factor 2 (Nrf2) in liver

A. It is localized in the cytoplasm and in the nucleus of the SECs of the liver in the control group. The staining intensity is moderate.

B. B: In the burned group, induction of Nrf2 positive cells was in cytoplasm of the SECs in individual hepatocytes. It was moderate to strong in the individually cells. In the burned group treated with melatonin, induction of Nrf2 positive cells was in the cytoplasm and in the nucleus in both hepatocytes and SECs. The number of HO-1 positive cells was higher than in the burned non treated. (Immunohistochemsitry, x 400).

transcriptional factor which induces pro-inflammatory cytokine expression [16]. Both TNF- $\alpha$ level and NF- $\kappa B$ expression in liver are reduced after melatonin administration [58]. These findings suggest that NF- $\mathrm{kB}$ activation promotes hepatic TNF- $\alpha$ production so TNF- $\alpha$ elevation further stimulates liver NF$\kappa \mathrm{B}$ transcriptional factor activation after burns. Both form a positive feedback loop and stimulate liver injury. After melatonin inhibition of the increased TNF- $\alpha$ level and NF- $\kappa$ B expression there is only restriction of liver injury evidenced by increased plasma transaminase (AST and ALT) activities and histological changes. Melatonin suppresses burn-induced oxidative injury, avoids thiol level depletion, increases antioxidant defense and restricts liver morphology disorders [32,39]. The morphological changes of inflammation such as edema, leukocyte infiltration and cell vacuolization are reduced in animals treated with melatonin [36].

Melatonin presents with other means to reduce inflammationinduced liver damage. Thus, it prevents translation of TNF- $\alpha$ binding of NF-kB and thereby, suppresses the production and release of proinflammatory mediators such as TNF- $\alpha$, other cytokines and adhesion molecules [59]. These data demonstrate that melatonin protection of burn-induced liver may be attributed by its antioxidant and ani-inflammatory properties.

The decreased TNF $\alpha$ level is accompanied by a marked rise of anti-inflammatory interleukin 10 (IL-10) in the animals, pretreated with melatonin $[55,56]$ and effect may be due to its anti-inflammatory properties [16]. Thus, melatonin affects the pro- /anti-inflammatory balance mitigating hepatic damage from uncontrollable inflammation. This may be one of the main mechanisms for the hepatoprotective effect of melatonin after burns.

Melatonin treatment may attenuate oxidative stress, inflammaton and delays deterioration of liver structure and function in rats during early response following burn trauma. Further studies would be directed to the possible useful applications of melatonin in specific, pharmacologically tested dosages to patients after severe acute thermal injury. If proven effective, melatonin may be an attractive therapy, since it is a natural, inexpensive, widely available, orally applicable, and relatively safe product. 


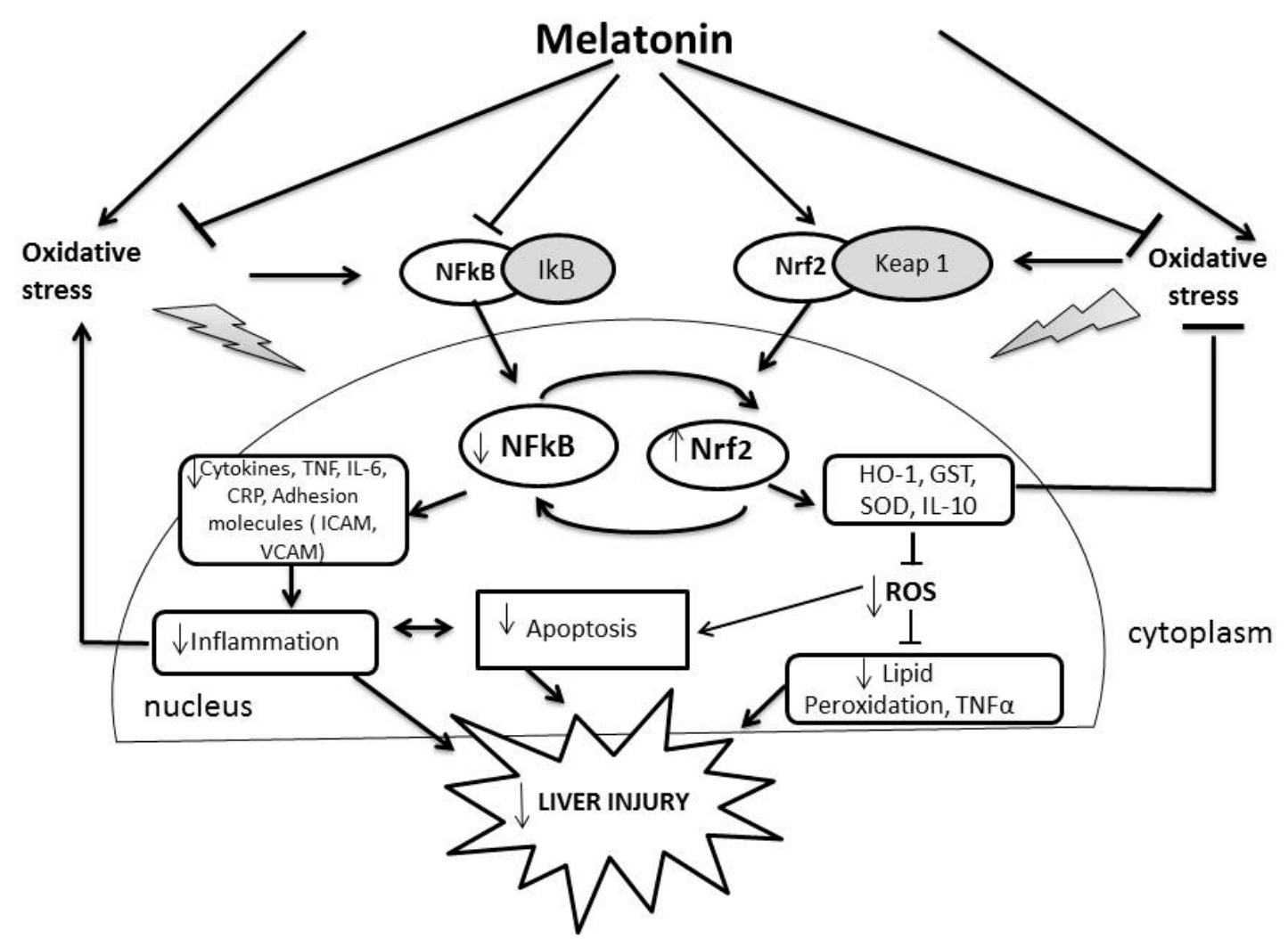

Figure 4: Schematic representation of the modulating effect of melatonin on Nf-kB and Nrf2 signaling pathways and its role in restricting the burninduced hepatic injury.

\section{References}

1. Agay D, Andriollo-Sanchez M, Claeyssen R, Touvard L, Denis J, Roussel $\mathrm{AM}$, et al. Interleukin-6, TNF-alpha and interleukin-1 beta levels in blood and tissue in severely burned rats. Eur Cytokine Netw.2008;19(1):1-7. doi: 10.1684/ecn.2008.0113.

2. Latha B, Babu M. The involvement of free radicals in burn injury: a review. Burns. 2001; 27(4):309-317.

3. Jeschke MG, Chinkes DL, Finnerty CC, Kulp G, Suman OE, Norbury WB, et al. Herndon DN. Pathophysiologic response to severe burn injury. Ann Surg. 2008;248(3):387-401. doi: 10.1097/SLA.0b013e3181856241.

4. Yang Q, Orman MA, Berthiaume F, Ierapetritou MG, Androulakis IP. Dynamics of short-term gene expression profiling in liver following thermal injury. J Surg Res. 2012;176(2):549-558. doi: 10.1016/j. jss.2011.09.052.

5. Sener G, Sehirli AO, Satiroglu H, Keyer-Uysal M, Yegen BC. Melatonin improves organ damages in rat model of thermal injury. Burns. 2002;28(5):419-425.

6. Reiter RJ, Tan, DX, Manchester LC, Qi W. Biochemical reactivity of melatonin with reactive oxygen and nitrogen species: A review of the evidence. Cell Biochem Biophys. 2001;34(2):237-256.

7. Rodriguez C, Mayo JC, Sainz RM, Antolín I, Herrera F, Martín V, et al Regulation of antioxidant enzymes: a significant role for melatonin. J Pineal Res.2004;36(1):1-9.

8. Radogna F, Diederich M, Ghibelli L. Melatonin: a pleiotropic molecule regulating inflammation. Biochem Pharmacol. 2010;80(12):18441852. doi: 10.1016/j.bcp.2010.07.041.

9. Maldonado MD, Murillo-Cabezas F, Calvo JR Lardone PJ, Tan DX, GuerreroJM, et al. Melatonin as pharmacologic support in burn patients: a proposed solution to thermal injury-related lymphocytopenia and oxidative damage. Crit. Care Med. 2007;35(4):1177-1185.

10. Korkmaz A, Reiter RJ, Topal T, Manchester LC, Oter S, Tan DX. Melatonin: an established antioxidant worthy of use in clinical trials. Mol Med. 2009;15(1-2):43-50. doi: 10.2119/molmed.2008.00117. doi: 10.2119/molmed.2008.00117.

11.El-Sokkary GH, Abdel-Rahman GH, Kamel ES. Melatonin protects against lead-induced hepaticand renal toxicity in male rats. Toxicology. 2005;213(1-2):25-33.

12. Chiu MH, Su CL, Chen CF Chen KH, Wang D, Wang JJ. Protective effect of melatonin on liver ischemia-reperfusion-induced pulmonary microvascular injury in rats. Transplant Proc. 2012;44(4):962-965. doi: 10.1016/j.transproceed.2012.01.097.

13. Jung KH, Hong SW, Zheng HM, Lee DH, Hong SS. Melatonin down regulates nuclear erythroid 2-related factor 2 and nuclear factor-kappa B during prevention of oxidative liver injury in a dimethylnitrosamine model. J Pineal Res. 2009; 47(2);173-183. doi: 10.1111/j.1600079X.2009.00698.x.

14. Ambriz-Tututi M, Rocha-Gonzalez HI, Cruz SL, Granados-Soto V. Melatonin: a hormone that modulates pain. Life Sci. 2009;84(1516):489-498. doi: 10.1016/j.lfs.2009.01.024. 
15. Berra B, Rizzo AM. Melatonin: circadian rhythm regulator chronobiotic, antioxidant and beyond. Clin Dermatol. 2009;27(2):20209. doi: 10.1016/j.clindermatol.2008.04.003.

16. Mauriz JL, Collado PS, Veneroso C, Reiter RJ, González-Gallego J. A review of the molecular aspects of melatonin's anti-inflammatory actions: recent insights and new perspectives. J Pineal Res. 2013;54(1):1-14. doi: 10.1111/j.1600-079X.2012.01014.x.

17. Tan DX, Manchester LC, Terron M P, Flores LJ, Reiter RJ. One molecule, many derivatives: a never-ending interaction of melatonin with reactive oxygen and nitrogenspecies? J Pineal Res. 2007;42(1):28-42.

18. Peyrot F, Ducrocq C. Potential role of tryptophanderivatives in stress responses characterized by the generationof reactive oxygen and nitrogen species? J Pineal Res. 2008;45(3):235-246. doi: 10.1111/j.1600-079X.2008.00580.x.

19. Reiter RJ, Tan DX, Jou MJ, Korkmaz A, Manchester LC, Paredes SD. Biogenic amines in the reduction of oxidative stress: melatonin and its metabolites. Neuro Endocrinol Lett. 2008;29(4):391-398.

20. Mart'nez-Cruz F, Osuna C, Guerrero JM. Mitochondrial damage induced by fetal hyper phenylalaninemia in the rat brain and liver: its prevention by melatonin, Vitamin E and Vitamin C. Neurosci Lett. 2006;392(1-2):1-4

21. Durante P, Romero F, P'erez M, Ch'avez M, Parra G. Effect of uric acid on nephrotoxicity induced by mercuric chloride in rats. Toxicol Ind Health. 2010;26(3):163-174. doi: 10.1177/0748233710362377.

22. Ochoa JJ, Díaz-Castro J, Kajarabille N, García C, Guisado IM, De Teresa $\mathrm{C}$, et al. Melatonin supplementation ameliorates oxidative stress and inflammatory signaling induced by strenuous exercise in adult human males. J Pineal Res. 2011;51(4):373-380. doi: 10.1111/j.1600079X.2011.00899.x.

23. Bergamini CM, Gambetti S, Dondi A, Cervellaci C. Oxygen reactive species and tissue damage. Curr Pharm Des. 2004;10(14):1611-1626.

24. Gałecka E, Mrowicka M, Malinowska K, Gałecki P. Role of free radicals in the physiological processes. Polski Merkuriusz Lekarski. 2008;24(143):446-448.

25. Afonso V, Champy R, Mitrovic D, Collin P, Lomri A. Reactive oxygen species and superoxide dismutases: role in joint diseases. Joint Bone Spine. 2007;74 (4):324-329.

26. Osburn WO, Wakabayashi N, Misra V, Nilles T, Biswal S, Trush MA, et al. Nrf2 regulates an adaptive response protecting against oxidative damage following diquat-mediated formation of superoxide anion. Arch Biochem Biophys. 2006;454(1):7-15.

27. Crespo I, Miguel BS, Laliena A Alvarez M, Culebras JM, González-Gallego $\mathrm{J}$, et al. Melatonin prevents the decreased activity of antioxidant enzymes and activates nuclear erythroid 2-related factor 2 signaling in an animal model of fulminant hepatic failure of viral origin. J Pineal Res. 2010;49(2):193-200. doi: 10.1111/j.1600-079X.2010.00787.x.

28. Morgan MJ, Liu ZG. Cross talk of reactive oxygen species and NF-kB signaling. Cell Res. 2011;21(1):103-15. doi: 10.1038/cr.2010.178.

29. Baeuerle PA, Baltimore D. NF-kappa: ten years after. Cell. 1996;87(1):13-20.

30. Luedde T, Schwabe RF. NF- $\kappa B$ in the liver--linking injury, fibrosis and hepatocellular carcinoma. Nat Rev Gastroenterol Hepatol. 2011;8(2):108-18. doi: 10.1038/nrgastro.2010.213.

31.Al-Ghoul WM, Abu-Shaqra S, Park BG, Fazal N. Melatonin plays a protective role in postburn rodent gut pathophysiology. Int J Biol Sci. 2010;6(3):282-293.
32. Bekyarova G, Tancheva S, Hristova M. Protective effect of melatonin against oxidative hepatic injury after experimental thermal trauma. Methods Find Exp Clin. Pharmacol. 2009;31(1):11-14. doi: 10.1358/ mf.2009.31.1.1338411.

33. Soybir G, Topuzlu C, Odabas O, Dolay KA, Bilir A, Koksoy F. The effects of melatonin on angiogenesis and wound healing. Surgery Today. 2003;33(12):896-901.

34. Dahiya P. Burns as a model of SIRS. Front Biosci (Landmark Ed). 2009;14:4962-4967.

35.Vinha PP, Martinez EZ, Vannucchi H, Marchini JS, Farina JA Jr, Jordao AA, et al. Effect of acute thermal injury in status of serum vitamins, inflammatory markers, and oxidative stress markers: preliminary data. J Burn Care Res. 2013;34(2): e87-91. doi: 10.1097/ BCR.0b013e31826fc506.

36. Bekyarova G, Tancheva S, Hristova M. The effects of melatonin on burn-induced inflammatory responses and coagulation disorders in rats. Methods Find Exp Clin Pharmacol. 2010;32(5):299-303. doi: 10.1358/mf.2010.32.5.1437717.

37. Park SW, Choi SM, Lee SM. Effect of melatonin on altered expression of vasoregulatory genes during hepatic ischemia/reperfusion. Arch Pharm Res. 2007;30(12):1619-1624.

38. Ashino T, Sugiuchi J, Uehara J, Naito-Yamamoto Y, Kenmotsu S, Iwakura $Y$, et al. Auranofin protects against cocaine-induced hepatic injury through induction of heme oxygenase-1. J Toxicol Sci. 2011;36(5):635-643.

39. Bekyarova G, Tzaneva M, Hristova M, Hristov K. Melatonin protection against burn-induced liver injury. A review. $\cdot$ Central European Journal of Medicine. 2014;9(1):148-158.

40. Niki E. Lipid peroxidation: physiological levels and dual biological effects. Free Radic Biol Med. 2009;47(5):469-484. doi: 10.1016/j. freeradbiomed.2009.05.032.

41. Piantadosi CA, Carraway MS, Suliman HB. Carbon monoxide, oxidative stress, and mitochondrial permeability pore transition. Free Radic Biol Med. 2006; 40(8):1332-1339.

42. Sandre C, Agay D, Ducros V, Van Uye A, Cruz C, Chancerelle Y, et al. Early evolution of selenium status and oxidative stress parameters in rat models of thermal injury. J Trace Elem Med Biol. 2004;17(4):313318.

43. Agay D, Anderson RA, Sandre C, Bryden NA, Alonso A, Roussel AM, et al. Alterations of antioxidant trace elements $(\mathrm{Zn}, \mathrm{Se}, \mathrm{Cu})$ and related metallo-enzymes in plasma and tissues following burn injury in rats. Burns. 2005;31(3):366-371.

44. Wang BH, Yu XJ, Wang D, Qi XM, Wang HP, Yang TT, et al. Alterations of trace elements $(\mathrm{Zn}, \mathrm{Se}, \mathrm{Cu}, \mathrm{Fe})$ and related metalloenzymes in rabbit blood after severe trauma. J Trace Elem Med Biol. 2007;21(2):102107.

45. Sass G, Barikbin R, Tiegs G. The multiple functions of heme oxygenase-1 in the liver. Z Gastroenterol. 2012;50(1):34-40. doi: 10.1055/s-00311282046.

46. Bekyarova G, Tzaneva M, Hristova M. Heme Oxygenase-1 Expresion in Gastric Mucosa and Liver after Burns: Preliminary Immunohistochemical Study. J Interdiscipl Histopathol. 2013;1(5):246-251.

47. Bekyarova G, Tzaneva M, Hristova M. Melatonin modulates the expression of Bcl-2 family proteins in liver after thermal injury in rats. Advances in Bioscience and Biotechnology. 2013;4(11A):41-44. 
48. Dulak J, Józkowicz A. Carbon monoxide -- a "new" gaseous modulator of gene expression. Acta Biochim Pol. 2003;50(1):31-47.

49. Ryter SW, Otterbein LE, Morse D, Choi AM. Heme oxygenase/carbon monoxide signaling pathways: regulation and functional significance. Mol Cell Biochem. 2002;234-235(1-2):249-263.

50. Chan K, Han XD, Kan YW. An important function of Nrf2 in combating oxidative stress: detoxification of acetaminophen. Proc Natl Acad Sci USA. 2001;98(8):4611-4616.

51. Farombi EO, Shrotriya S, Na HK, Kim SH, Surh YJ. Curcumin attenuates dimethylnitrosamine-induced liver injury in rats through Nrf2mediated induction of heme oxygenase-1. Food Chem Toxicol. 2008;46(4):1279-1287.

52. Miller DM, Singh IN, Wang JA, Hall ED. Administration of the Nrf2-ARE activators sulforaphane and carnosic acid attenuates 4-hydroxy-2nonenal-induced mitochondrial dysfunction ex vivo. Free Radic Biol Med. 2013;57:1-9. doi:10.1016 /j.freeradbiomed. 2012.12.011.

53. Jaiswal AK. Nrf2 signaling in coordinated activation of antioxidant gene expression. Free Radic Biol Med. 2004;36(10):1199-1207.
54. Finnerty CC, Herndon DN, Przkora R, Pereira CT, Oliveira HM, Queiroz DM, et al. Cytokine expression profile over time in severely burned pediatric patients. Shock. 2006;26(1):13-19.

55. Despond O, Proulx F, Carcillo JA, Lacroix J. Pediatric sepsis and multiple organ dysfunction syndrom. Curr Opin Pediatr. 2001;13(3):247-253.

56. Schneider CP, Schwacha MG, Chaudry IH. The role of interleukin-10 in the regulation of the systemic inflammatory response following trauma-hemorrhage. Biochim Biophys Acta. 2004;1689(1):22-32.

57. Sabat R. IL-10 family of cytokines. Cytokine Growth Factor Rev. 2010;21(5):315-324. doi: 10.1016/j.cytogfr.2010.11.001.

58. Bekyarova G, Apostolova M, Kotzev I. Melatonin protection against burn-induced hepatic injury by down-regulation of nuclear factor kappa B activation. Int J Immunopathol Pharmacol. 2012;25(3):591596.

59. Horton JW. Free radicals and lipid peroxidation mediated injury in burn trauma: the role of antioxidant therapy.Toxicology. 2003;189(12):75-78. 